403 TGW participated; 52.1\% were aged 18-33 years; mean 34.4 (SD \pm 9.6 ); $57.6 \%$ had $\geq 12$ years of formal education; $70 \%$ identified as black/mixed race; $25.8 \%$ declared sex work as main source of income and $48.1 \%$ that their monthly income was below or equal to the Brazilian minimum wage. Prevalence of chlamydia and gonorrhea was, respectively, $10.2 \%$ (41/403) and 8.2\% (33/403); coinfection was $2.4 \%(10 / 403)$. Most TGW testing positive had anal infections: $90.2 \%$ for chlamydia and $66.7 \%$, gonorrhea. Prevalence of chlamydia or gonorrhea was slightly higher among individuals living with HIV (18\% vs $15 \%$ among HIV-negative individuals, OR 1.21; 95\% CI 0.67 - 2.19). After adjusting for schooling, sex work as main source of income, and monthly income, young age remained a risk factor for a chlamydia (aOR 2.78; 95\% CI 1.34 - 5.74) and gonorrhea (aOR 2.21; 95\% CI 1.02 - 4.79) diagnosis.

Chlamydia and gonorrhoea prevalence is high among Brazilian TGW, especially amongst the young. Comprehensive care and prevention programs, including sexual education and screening polices directed at TGW are urgently needed to reduce STI burden and to interrupt STI/HIV transmission.

\section{6 'IF THEY ASK, I WILL TELL THEM': ATTITUDES TOWARDS ACCESSING SEXUAL HEALTHCARE AMONG HETEROSEXUAL-IDENTIFYING MSM IN ENGLAND}

${ }^{1} \mathrm{~T}$ Curtis*, ${ }^{1} \mathrm{C}$ Mercer, ${ }^{1} \mathrm{~N}$ Field, ${ }^{2} \mathrm{~L}$ McDonagh. ${ }^{1}$ University College London, Institute for Global Health, London, UK; ${ }^{2}$ University College London, NIHR Health Protection Research Unit in Blood Borne and Sexually Transmitted Infections, London, UK

\subsection{6/sextrans-2021-sti.139}

Background STI/HIV testing is lower among heterosexual-identifying men who have sex with men (heterosexual-MSM) than bisexual or gay men. We aimed to understand attitudes towards sexual healthcare among heterosexual-MSM in England, to improve service design and uptake among this overlooked population.

Methods Semi-structured individual interviews were conducted with 15 heterosexual-MSM in England in January-March 2020. Participants ranged in age from 22-69 years. All but one reported current or previous relationships with women. Data were analysed using an inductive thematic analysis.

Results Frequency of STI/HIV testing varied widely between participants, reflecting how some men felt they lacked sufficient or accurate information about testing guidelines and options, including the possibility of home-sampling/testing. Among men with female partners, concern for the health and wellbeing of these partners was a motivator for testing. However, privacy and discretion were important factors in the use of home-sampling/testing kits for men living with female partners or family; their ability to use these services was limited when their privacy needs were not accommodated. Their heterosexual identity meant some felt services intended for gay and bisexual men were not appropriate for them. If asked by sexual health clinicians, most heterosexual-MSM interviewed reported feeling comfortable disclosing the sex they have with men, describing the impersonal nature of consultations and perceptions of non-judgement and discretion as facilitators for disclosure. However, this comfort with disclosure did not always extend to GPs, due to fears their behaviour would be exposed to others.

Conclusion For the heterosexual-MSM in this study, privacy and discretion were of utmost importance. These must be guaranteed by sexual healthcare services, whether in-clinic or home-sampling/testing, to appeal to MSM regardless of their personal circumstances. Trust in clinician confidentiality and non-judgement facilitate disclosure. Further work is needed to identify ways for sexual health services to appeal to, and reach, this population.

\section{Female reproductive health for STIs and HIV}

\subsection{HIGH BURDEN OF REPRODUCTIVE TRACT INFECTIONS AND POOR SEXUAL AND REPRODUCTIVE HEALTH IN PREGNANCY AND POSTPARTUM IN PAPUA NEW GUINEA}

\begin{abstract}
${ }^{1,2} \mathrm{M}$ Scoullar*, ${ }^{1} \mathrm{P}$ Boeuf, ${ }^{1} \mathrm{E}$ Peach, ${ }^{1} \mathrm{E}$ Kearney, ${ }^{1} \mathrm{R}$ Fidelis, ${ }^{1} \mathrm{~K}$ Tokmun, ${ }^{1} \mathrm{P}$ Melepia ${ }^{2,3,4} \mathrm{C}$ Bradshaw, ${ }^{5} \mathrm{~A}$ Elijah, ${ }^{3} \mathrm{G}$ Fehler, ${ }^{6} \mathrm{P}$ Siba, ${ }^{7} \mathrm{~S}$ Erskine, ${ }^{7} \mathrm{E}$ Mokany, ${ }^{1} \mathrm{E}$ Kennedy, ${ }^{1} \mathrm{~A}$ Umbers, ${ }^{1,8,9} \mathrm{~S}$ Luchters, $1,2,4,6 \mathrm{~L}$ Robinson, ${ }^{4} \mathrm{~N}$ Wong, ${ }^{6,10} \mathrm{~A}$ Vallely, ${ }^{10} \mathrm{~S}$ Badman, ${ }^{10,11} \mathrm{~L}$ Vallely, $1,2,4 \mathrm{~F}$ Fowkes, ${ }^{1} \mathrm{C}$ Morgan, ${ }^{6} \mathrm{~W}$ Pomat, ${ }^{1,2,4} \mathrm{~B}$ Crabb, ${ }^{1,2,4} \mathrm{~J}$ Beeson, H Study Team. 'Burnet Institute, Australia; 'University of Melbourne, Melbourne, Australia; ${ }^{3}$ Melbourne Sexual Health Centre, Alfred Hospital, Australia; ${ }^{4}$ Monash University, Melbourne, Australia; ${ }^{5}$ School of Medicine and Health Sciences, University of Papua New Guinea, Papua New Guinea; ${ }^{6}$ Papua New Guinea Institute of Medical Research, Papua New Guinea; ${ }^{7}$ SpeeDx Pty Ltd, Australia; ${ }^{8} 8$ Department of Population Health, Aga Khan University, Nairobi, Kenya; International Centre for Reproductive Health, Department of Public Health and Primary Care, Ghent University, Belgium; ${ }^{10}$ The Kirby Institute, University of New South Wales, Australia; ${ }^{11}$ Australian Institute of Tropical Health and Medicine, James Cook University, Townsville
\end{abstract}

\subsection{6/sextrans-2021-sti.140}

There is a pressing need for detailed knowledge of the range of pathogens, extent of co-infection and clinical impact of reproductive tract infections (RTIs) among pregnant women. We present prevalence and correlates of RTIs (Mycoplasma genitalium, Chlamydia trachomatis, Neisseria gonorrhoeae, Trichomonas vaginalis, Treponema pallidum subspecies pallidum, bacterial vaginosis and vulvovaginal candidiasis) in a longitudinal study of women in pregnancy and postpartum in Papua New Guinea (PNG).

699 pregnant women were recruited at their first antenatal clinic visit and followed up at childbirth, one, six and twelve months postpartum. Self-collected vaginal swabs were tested for M.genitalium using real-time $\mathrm{PlexPCR}^{\circledR}$ (SpeeDx) which provides results for five point mutations associated with macrolide resistance. Urine samples or vaginal swabs were tested for C.trachomatis, N.gonorrhoea and T.vaginalis using GeneXpert. A vaginal smear was examined for BV and VVC. Routine antenatal services tested for syphilis using Alere DetermineTM Syphilis.

Most pregnant women $(74.1 \%)$ had at least one RTI, with a curable current sexually-transmitted infection (STI) detected in $37.7 \%$. We found $M$. genitalium, an emerging pathogen in PNG, in $12.5 \%$ of pregnant women, decreasing to $6.1 \%$ at six months postpartum, with no evidence of macrolide resistance. Prevalence of other curable STIs (C. trachomatis, N. gonorrhoeae and $\mathrm{T}$. vaginalis) were all high in in pregnancy 
$(19.1 \%, \quad 5.5 \%$ and $20.1 \%$ respectively), with prevalence decreasing immediately postpartum but rising again by 12 months postpartum. Clinical symptoms missed at least $75 \%$ of infections and there was little use of contraception; $98.4 \%$ report never having used barrier contraception.

This study highlights a high prevalence of a RTIs in pregnancy and postpartum. Most of these infections are curable when diagnosis is made available and syndromic management alone is insufficient. This high prevalence of disease negatively affects sexual and reproductive health and these findings have important public health implications in PNG and the region.

\subsection{EPISODIC PREP USE AMONG YOUNG WOMEN IN SIAYA COUNTY, KENYA}

A Corneli ${ }^{*}$, B Perry, D Ochieng Ngoje, N Molokwu, K Agot. Duke University, Durham, USA

10.1136/sextrans-2021-sti.141

Introduction The DREAMS Initiative provides PrEP to young women (YW) in Siaya County, Kenya, to combat the region's high HIV incidence. Women must take PrEP consistently for three weeks for full protection; episodic use may diminish PrEP's effectiveness. We describe findings on episodic PrEP use from participatory-based research to identify multi-level factors influencing YW's PrEP use.

Methods Using photovoice, YW and four groups in their social ecology_female peers, male peers/partners, family, and community members, including healthcare providers-participated in four iterative activities. First, participants photographed factors influencing YW's PrEP persistence. Second, participants discussed photographs with their own group (e.g., YW discussed with other YW). Third, YW and members in other groups (e.g., female peers) discussed each other's photographs and answered questions on themes from previous discussions. Fourth, YW shared individual experiences on themes during in-depth interviews. All discussions/interviews were audiorecorded, simultaneously translated/transcribed, and analyzed using applied thematic analysis. We focus on data from YW and female peers.

Results During group discussions, YW and female peers $(n=27)$ explained that YW perceive male partners as the primary source of HIV risk. As a result, YW commonly stop taking PrEP when partners are away for a few days to several weeks; they start taking PrEP again the day their partners return. Participants stressed that taking PrEP consistently for three weeks may be difficult for some because YW are unaware of their partners' return date and husbands' unwillingness to delay sex. Among YW interviewed $(n=18)$, nearly all their partners traveled, and about one-third reported stopping PrEP during their partners' absences. Reasons two-thirds continue include not knowing partners' sexual behaviors when traveling, and because PrEP provides HIV protection in case of accidents or rape.

Conclusion Counseling on PrEP persistence during partners' short absences is essential for $\operatorname{PrEP}$ to be most effective among YW in Kenya.

\subsection{HIGH PREVALENCE OF SEXUALLY TRANSMITTED INFECTIONS AMONG YOUNG AFRICAN WOMEN IN REACH STUDY OF ORAL EMTRICITABINE-TENOFOVIR AND DAPIVIRINE VAGINAL RING}

${ }^{1} \mathrm{C}$ Akello*, ${ }^{2} \mathrm{D}$ Szydlo, ${ }^{3} \mathrm{P}$ Macdonald, ${ }^{4} \mathrm{~B}$ Siziba, ${ }^{5} \mathrm{~T}$ Palanee-Phillips, ${ }^{6} \mathrm{M}$ Garcia, ${ }^{6} \mathrm{~T}$ McClure, ${ }^{7} \mathrm{~S}$ Johnson, ${ }^{7} \mathrm{~L}$ Levy, ${ }^{8} \mathrm{~K}$ Ngure, ${ }^{3} \mathrm{G}$ Nair, ${ }^{9} \mathrm{EL}$ Soto-Torres, ${ }^{2} \mathrm{RE}$ Brown, ${ }^{10,11} \mathrm{C}$ Celum, 3,11,12EJ Balkus. 'Makerere University Johns Hopkins Research Collaboration, Kampala, Uganda; ${ }^{2}$ Statistical Center for HIVIAIDS Research and Prevention, Fred Hutchinson Cancer Research Center, Seattle, USA; ${ }^{3}$ Desmond Tutu HIV Centre, University of Cape Town, Emavundleni Research Centre, CapeTown, South Africa; ${ }^{4}$ University of Zimbabwe-Clinical Trials Research Centre, Harare, Zimbabwe; ${ }^{5}$ Wits Reproductive Health and HIV Institute, Faculty of Health Sciences, University of the Witwatersrand, Johannesburg, South Africa; ${ }^{6}$ Family Health International 360 (FHI360), Durham, USA; ${ }^{7}$ Family Health International 360 (FHI360), Washington DC, USA; ${ }^{8}$ Jomo Kenyatta University of Agriculture and Technology, College of Health Sciences, Nairobi, Kenya; ${ }^{9}$ National Institute of Allergy and Infectious Diseases, National Institutes of Health, Bethesda, USA; ${ }^{10}$ Department of Medicine, University of Washington, Seattle, USA; ${ }^{11}$ Department of Global Health, University of Washington, Seattle, USA; ${ }^{12}$ Department of Epidemiology, University of Washington, Seattle, USA

\subsection{6/sextrans-2021-sti. 142}

Background With antiretroviral (ARV)-based HIV prevention, the hidden epidemic of sexually transmitted infections (STIs) is being uncovered. Most STIs are asymptomatic and not treated by syndromic case management, the standard of care in Africa. There are limited data on STI prevalence among African adolescent girls and young women (AGYW) who are at risk for sequelae from untreated STIs.

Methods MTN-034/REACH is a randomized, open label, crossover study assessing the safety of and adherence to the dapivirine vaginal ring and oral emtricitabine-tenofovir in Cape Town and Johannesburg, South Africa, Kampala, Uganda and Harare, Zimbabwe. Sexually active HIV-negative AGYW ages 16-21 years were enrolled and tested for Chlamydia trachomatis/Neisseria gonorrhoeae (CT/GC) by nucleic acid amplification, Trichomonas vaginalis (TV) by rapid test, and syphilis by serology. AGYW with positive test results received treatment. Descriptive statistics were used to summarize STI prevalence at baseline and selected characteristics by STI status.

Results Among 247 AGYW enrolled in REACH, 34\% were 16-17 years old, $87 \%$ single, and the median number of sexual partners in the prior 3 months was 1 (IQR 1-2). At enrollment, 87 (35\%) tested positive for any STI with 17 (7\%) with >1 STI: 71 (29\%) for CT, 21 (8.5\%) for GC, 12 $(4.9 \%)$ for TV and 6 (2.4\%) for syphilis. Of AGYW diagnosed with an STI, 90\% were asymptomatic. The prevalence of any STI varied by site: $47 \%$ in Cape Town, $37 \%$ in Johannesburg, $32 \%$ in Kampala, and $25 \%$ in Harare. STI prevalence was higher among 18-21 year olds versus 16-17 year olds $(37 \%$ vs $32 \%)$.

Conclusion STI prevalence among AGYW initiating oral or topical PrEP was alarmingly high and most were asymptomatic, highlighting the limitations of syndromic STI management. There is an urgent need and imperative to provide diagnostic STI testing to AGYW with the delivery of HIV prevention methods. 